\title{
Design of Scheduling Algorithm for QoS Management on WiMAX Networks
}

\author{
Hattab Guesmi $^{1,2, *}$, Sassi Maaloul ${ }^{1}$, Rached Tourki ${ }^{1}$ \\ ${ }^{1}$ Laboratory of electronics and micro-electronics, Monastir university, Tunisia \\ ${ }^{2}$ Faculty of sciences, Jazan university, KSA
}

\begin{abstract}
The apparition of high speed networks and the fast evolution of communication offer currently an unlimited access to an important number of applications. The applications constraints (videoconferencing, communications, VoIP, applications multimedia, etc...) are more complex for wireless systems because they require more constraints: high throughput, low packet loss, low latencies and gigue delays. All present flows in the WiMAX network must share the same medium capacity. Therefore, WiMAX is required to fulfill QoS requirements of any application and information passing over the network. Several mechanisms for QoS management have been studied, among these mechanisms there is an appropriate scheduler implementation for packets carried on WiMAX network which can increase QoS achievement possibility.WiMAX module used in our simulation deploys scheduling algorithms (FIFO, FQ, DRR, and WFQ) to deal with packets transmission. This paper is aimed at evaluating these algorithms in relation with WiMAX network performance. Performance metrics reported in this work are packet loss, throughput, and average delay. This paper tries to design an architecture which is able to convey the data belonging to various classes of traffic that guarantee their respective needs. Simulation results can show the performance of scheduling schemes used in this architecture.
\end{abstract}

Keywords IEEE802.16, Quality of Service, WFQ Scheduler, WiMAX, Network Simulator-2

\section{Introduction}

WiMAX is the acronym of Worldwide Interoperability for Microwave Access. It is a solution for the wireless Metropolitan Area Network (WMAN). It is based on Institute of Electrical and Electronics Engineers (IEEE) 802.16 standard[1],[2]. It offers the possibility to cover long distances with a high throughput. This standard authorizes the use of the Internet Protocol (IP) in a larger radius, based on the broadband wireless access (BWA) systems[1], which allows to achieve links between different subscribers station (SSs) and base station (BS), designed to provide access services network. To manage the various types of applications, standard 802.16 is subdivided in several sub-layers. Those make it possible to convert information so as to make them exploitable. The physical layer details the support of transmission and bandwidth. It is generally regarded as being in the lower part of physical layer OSI. At the top of the physical layers, the function charged to provide a service to subscriber intervene. These functions are gathered within the MAC layer. The protocol operates in this layer between the base station and subscriber station, and manages access to shared radio channel. Specifically, it defines

* Corresponding author:

hattab.guesmi@fsm.rnu.tn (Hattab Guesmi)

Published online at http://journal.sapub.org/computer

Copyright (C) 2011 Scientific \& Academic Publishing. All Rights Reserved how and when a base station or subscriber can initiate a transmission on the channel. The IEEE 802.16 standard has the advantage of allowing a wireless connectivity between base station and thousands of subscribers without requiring direct visual line. Also, it can deliver information by using several techniques of modulations. These techniques are used by ascending order of throughput: Binary Phase-Shift Keying, Quadrature Phase-Shift keying and Quadrature Amplitude Modulation. This makes it possible to bring closer and increase the number of carrier waves in a range frequency without having interferences between them.

This technology of transport permits to distribute IP services over variety of applications. Flows generated by these applications have several features: flow type (voice, video, data...), exchanged information volume, length of interactions, security, availability etc. These flows have also different needs of QoS requiring a treatment according to their constraints. The remainder of this paper is organized as follows: In section II, we submit an overview of related works. Section III presents an overview of the QoS management in IEEE 802.16 wireless MAN. Section IV describes the scheduling mechanism to support QoS. The simulation environment and the results analysis are presented in section V. Finally, Section VI contains some conclusions.

\section{Related Works}


In the recent years, many proposals were enriching the literature of QoS support for wireless networks. Most of the researches are based on IEEE 802.16 standard. An overview of some of these approaches can be found in[3-8]. They can be divided into categories to define the requirements, such as parameters that indicate quality of service and mechanisms that act over these parameters. In the first category QoS analyzes in WiMAX networks. Rohit and Mohammad[3] studied the quality of service architecture and analyzed parameters that indicate quality of service, such as, throughput, packet loss, average jitter and average delay. They concluded that VOIP traffic can be served with UGS service flow and that the rtPS service flow is designed for applications such as streaming audio and streaming video. Aymen and Loutfi[4] work consists in the addition of the QoS classes as well as the QoS management requirements, unicast and contention request opportunities mechanisms, and scheduling algorithms for the UGS, rtPS and BE QoS classes. This work is conducted to show that the behavior of UGS, rtPS, and BE schedulers fits with the QoS specifications of the IEEE 802.16 standard. Another category which is based on the scheduling algorithms, many algorithms are specifically designed for WiMAX. The major aim of the study of Jin-Cherng et al.[5] a description and performance evaluation of scheduling algorithms. They show through simulation the best performance for different queue scheduling in the network. It seems that WFQ are not the best selection in performance, even though WFQ give weight to control bandwidth allocation and priority. If the system needs the best throughput performance in this networks transport environment, RIO scheduling scheme is the best scheduling. As well, Najah et al.[6] present a performance study of uplink scheduling algorithms in point-to-multipoint WiMAX networks. They concluded that there is no single scheduling scheme that provides the desired performance with respect to all QoS requirements and characteristics of the IEEE802.16 MAC layer. A scheduling algorithm needs to be selected based on the requirements and traffic profiles of the network.

In our study, we focus our work on the implementation of various scheduling algorithms for IEEE 802.16 wireless MAN using network simulator, where a brief description and performance evaluation to provide the better packet scheduling to various application. In this setting, we implemented architecture able to transport the data belonging to several classes of traffic, to provide better packet scheduling to each application. We study and analyze the behavior of each scheduling algorithms in time and when the number of nodes increases. Then, we evaluate the algorithms using parameters that indicate quality of service such as: average delay, average jitter, packet loss and throughput, for all service classes.

\section{The Overview of the QoS Management}

\subsection{IEEE 802.16 Service Classes}

The classification mechanism identifies and separates different traffics into flows or group of flows. Therefore each flow or group of flows can be handled differently. Application traffic is identified by the classification mechanism and it is forwarded to the appropriate queue awaiting service from other mechanism such as traffic shaping and packet scheduling. The granularity level of the classification mechanism can be per-user, per-flow or per-class depending on the type of QoS provided services. To identify and classify the traffic, the traffic classification mechanism requires some forms of tagging or marking of packets. The 802.16d MAC provides QoS differentiation for different types of applications that might operate over 802.16 networks that define the following types of services[9, 10, 11, 12]:

- Unsolicited Grant Service is designed to support real-time applications (with strict delay requirements). The transmission must be carried out with fixed size data packets to regular interval constant bit rate (CBR) or fixed throughput connections such as voice over IP (VoIP) and E1/T1 lines. UGS connections never request bandwidth. It is given periodic bandwidth. This service provides guarantees on throughput, latency and jitter.

- Real-time Polling Service this second class of service is designed to support real-time applications (with less stringent delay requirements) that generate variable size data packets to regular interval variable bit rate (VBR) such as MPEG video, streaming video and audio. rtPS connections are required to notify the BS of their current bandwidth requirements. It provides guarantees on throughput and latency, but with greater tolerance on latency relative to UGS.

- Non-real-time Polling Service this class of service allows the transmission of flows with flexible delay such as File Transfer Protocol (FTP). It provides guarantees in terms of throughput only and it is therefore suitable for mission critical data application. These applications require minimum throughput.

- Best Effort is designed for the application which does not have any guarantee in terms of throughput or packet delay, such as internet navigation, telnet, HTTP... It's used more and more in the web services. The BE applications receive the residual bandwidth after the bandwidth is allocated to the connections of the previous three service classes.

\subsection{QoS management}

QoS corresponds to all mechanisms that allow a network to distribute equitably and according to requirements of applications all the resources offered by networks, to provide the need quality. Also, it can be characterized by different performance criteria that include basic availability, the loss rate, throughput, average delay, security, etc... To provide an efficient level of QoS support, many mechanisms can be managed tightly coupled and plays an essential role in determining the network performance[11, 12, 13, 14, 15]:

- Admission control is a network Quality of Service 
(QoS) procedure. Admission control determines how bandwidth and latency are allocated therefore need to be implemented between network SS and BS to control the traffic entering the network. The role of CAC is to control the number of connection flows into the network. A new connection request is progressed only when sufficient resources are available at each successive network element to establish the connection through the whole network based on its service category, traffic contract and QoS, while the agreed QoS of all existing connections are still maintained. Admission control is useful in situations where a certain number of connections may all share a link, while an even greater number of connections cause significant degradation in all connections to the point of making them all useless such as in congestive collapse.

- Packet scheduling refers to the decision process used to choose which packets should be served, it is the process of resolving contention for bandwidth. The target of a scheduling algorithm has to determine the allocation of bandwidth among the users and their transmission order. One of the most important tasks of a scheduling scheme resides in satisfying the Quality of Service (QoS) requirements of its users while efficiently utilizing the available bandwidth.

- Buffer management refers to any particular discipline used to regulate the occupancy of a particular queue where packets may be held (or dropped). Buffer is set to improve link utilization and system performance, but it also increases packet's queue delay. With the increase of user demands for service quality, providing stable and low delay was the primary requirement of real-time services. The most important and easy controls part of total delay is queue delay. So how to set the capacity of the buffer, how to control efficiently buffer length while network circumstance is dynamic and how to achieve the tradeoff between throughput and queue delay. These are the important problems to be solved in buffer management and QoS control of whole networks.

Figure 1 shows the scheme of the QoS management mechanism. At the beginning of transmission, each SS through a phase of registration (connect) with the BS. Thus, the BS allocates a CID to each connection and the backup settings according to their service class. Uplink packet processing is handled by the Base Station (BS) through signaling process to the Subscriber Station (SS). Each SS has a scheduler to decide which packet will be sent from the queue to the network in the proper time interval as defined in Uplink Map Message (UL-MAP) sent by BS in subsidies allocated data in BS. Two types of scheduling are localized in the BS: Downlink scheduler determines which packets of the upper layer will be next downlink. This decision is based on the QoS requirements and situation of the queue. Uplink scheduler decides which SS can transmit next uplink, and the number of slots that can use SS. This decision is based on the quality requirements and service demands of bandwidth sent by the SS.

\section{Packet Scheduling}

The fundamental problem solved by a scheduling algorithm running at a router is to answer the following question: "Which packet gets sent out next, and when?" The choice of which flow is the next one to use an outbound link helps apportion bandwidth between flows. At the same time, a scheduling algorithm can also decide when to send any given packet (or flow's packet), a choice that can help guarantee (or bound) packet latencies through the router. The idea of scheduling is to adapt the policy of transmission of packets in buffers, according to the requirements of QoS for flows. Scheduling has a significant impact not only on the average delay but also on the buffer size. Scheduling is used to control the resources distribution between the classes of service. Many legacy scheduling algorithms, able to provide certain guaranteed QoS, have been developed for wireless networks. The characteristics of wireless communication pose special problems that do not exist in wireline networks. They include[14, 15]:

- High error rate and bursty errors,

- Location-dependent and time-varying wireless link capacity,

- Scarce bandwidth,

- Users mobility,

- Power constraint of the mobile hosts.

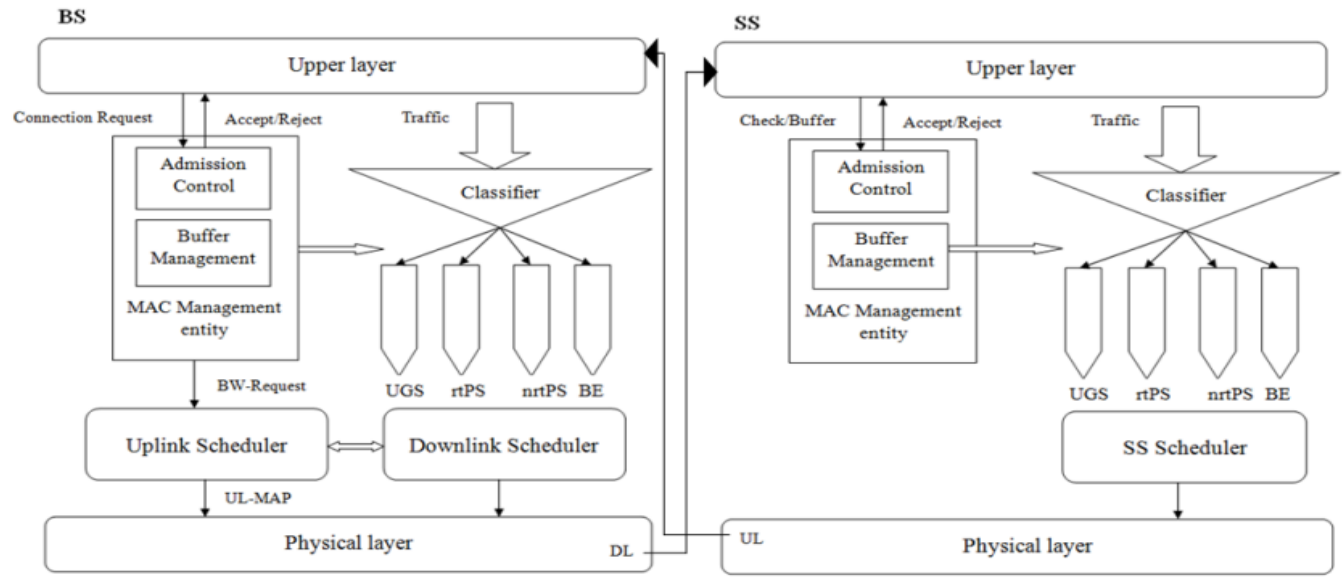

Figure 1. QoS management mechanism 
All of the above characteristics make developing efficient and effective scheduling algorithms for very challenging wireless networks. WiMAX networks provide services for heterogeneous classes of traffic with different quality of service (QoS) requirements. Currently, there is an urgent need to develop new technologies for providing QoS differentiation and guarantees in WiMAX networks. Among the most important technical issues that need to be resolved, there is packet scheduling in WiMAX networks. In this sub-section, we assess proposed scheduling algorithms for QoS support in WiMAX networks thoroughly with respect to the characteristics of the IEEE 802.16 MAC layer and PHY layer[3, 4, 5, 6, 7 and 8]. With respect to the nature of scheduling, algorithm mechanism can be classified as per-flow, per-class and per-packet scheduling algorithms. Representative schemes in each of these categories will be discussed after. So far, several proposals have been insightful in the present literature of QoS support for wireless networks. We can find several such as FIFO, RR, CBQ, FQ, DRR, WRR and WFQ. The algorithms implemented, compared and performance evaluated in this architecture are FIFO, Deficit Round Robin, Fair Queuing and Weighted Fair Queuing.

\section{Experimental Results}

\subsection{Structure Simulated Environment}

To analyze QoS management on Wimax networks it is necessary to study real life scenarios. The network topology described in the figure 2 is used to simulate the proposed scheduling algorithms in order to evaluate the architecture performance that supports QoS. It consists in two parts: the first part defines the sources or the subscriber stations and the second part defines the base station. The scheduling algorithms show interesting results when they are studied under different mix of traffic. Thus, we have created four sources emitting each one a specific type of traffic. The type of traffic based on UDP protocol uses the CBR applications is assigned to the UGS class. The second source is the rtPS class that uses the VBR applications based on the UDP protocol. The third source defines the nrtPS class that uses the FTP applications based on TCP protocol. Finally the fourth source uses the FTP applications based on TCP protocol assigned for the BE class. The simulation time is fixed to 100 seconds to analyze and evaluate each algorithm to guarantee the QoS for all types of applications. During the simulation we increase the number of sources (nodes) to evaluate the behavior of each algorithm also when the network is congested. The implemented model is based on the NIST[12, 13] (National Institute of Standards and Technology) model over the network simulator NS-2.33. This module offers several features[15]: Wireless MAN-OFDM physical layer with configurable modulation, Time Division Duplexing (TDD), management messages to execute network entry (without authentication), default scheduler providing round robin uplink allocation to registered, mobile Stations (MSs) according to bandwidth requested, IEEE 802.16e extensions to support scanning and handovers and fragmentation and reassembly of frames. It is important to note that many components are not defined in the standard such us: Wireless MAN-OFDMA, Frequency Division Duplexing (FDD), Service Flow and QoS scheduling.

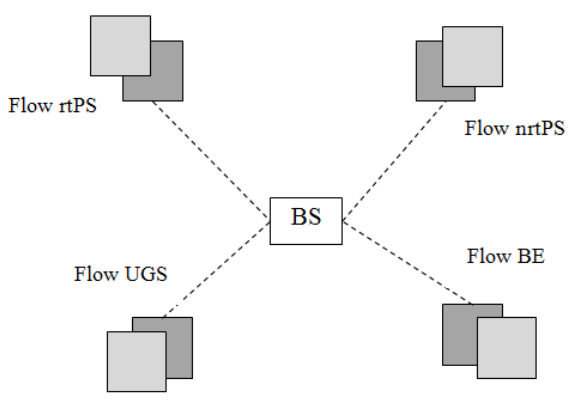

Figure 2. Topology of the simulated network

In the current implementation, our contribution consist in implement some scheduling schemes in WiMAX model to simulate real network and conclude from the simulation results to show QoS requirement. Therefore, we assume that subscriber stations cooperate with different types of traffic service and achieve several scheduling algorithms (DropTail, FQ, DRR and WFQ) in NS2. The main simulation parameters are listed in Table 1.

Table 1. Parameter simulation

\begin{tabular}{|c|c|}
\hline Parameters & Values \\
\hline Frequency bandwidth & $5 \mathrm{MHZ}$ \\
\hline Modulation Type & OFDM_QPSK \\
\hline Length of frame & $5 \mathrm{~ms}$ \\
\hline Time simulation & $100 \mathrm{~s}$ \\
\hline Routing Protocol & OSDV \\
\hline Mean length of packet & 1024 bytes \\
\hline Number of nodes & 24 \\
\hline
\end{tabular}

\subsection{Simulation Results}

These simulations analyze the parameters of QoS which are strongly related to the applications performances. The same architecture, the same parameters and the same traffics are used for various scheduling algorithms. During simulations we vary the numbers of Subscriber Station (SS) attached to each class of service.

$\rightarrow$ The performance evaluation of each class of service

A series of simulations have been done where the nodes number is incremented each time, to see the behavior of the algorithms and its influence on the degradation of the QoS related to each application. Figure 3 to 14 show the result for QoS parameters (delay, jitter ...) variation of UGS, rtPS, nrtPS, and BE service classes depending on the number of nodes.

The delay for different classes of service rises to the peak, this increase is due to queues saturation. If the number of nodes exceeds 16 , the average time decreases, this reduction reflects the reduction in the number of packets received after the onset of congestion in queues for rtPS, nrtPS and BE 
flows, as shown in Figure 3. This simulation presents that average delay for UGS application is guaranteed using the WFQ algorithms and the minimum delay is obtained using the same algorithm for all kinds of applications.

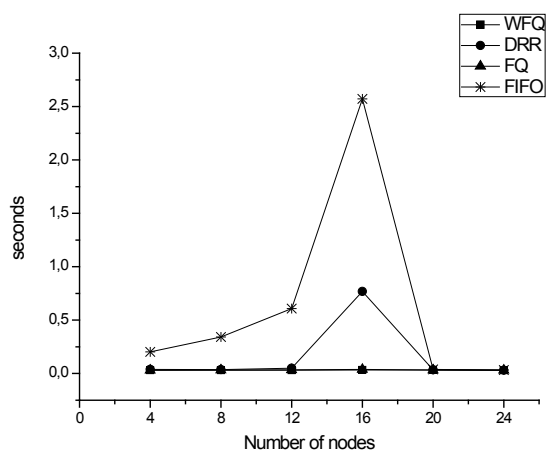

Figure 3. Average Delay for UGS

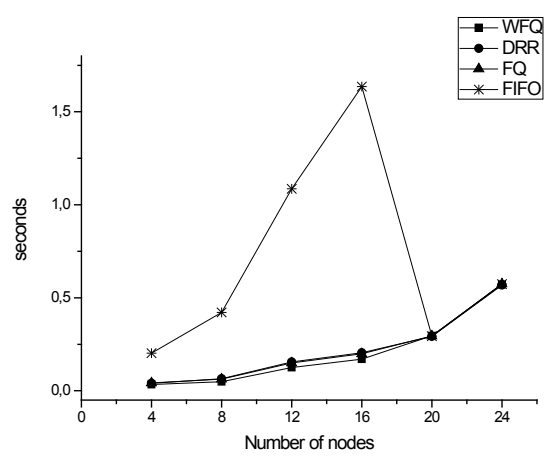

Figure 4. Average Delay for rtPS

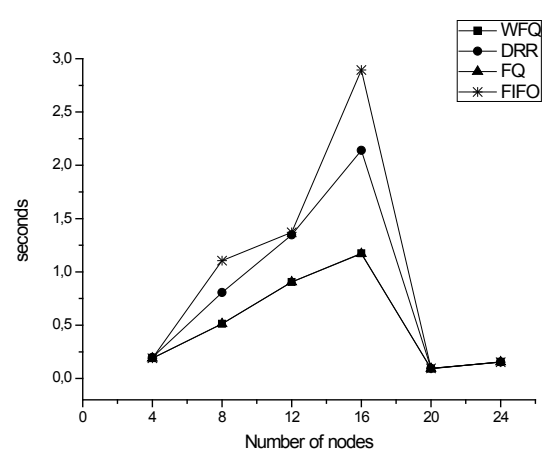

Figure 5. Average Delay for nrtPS

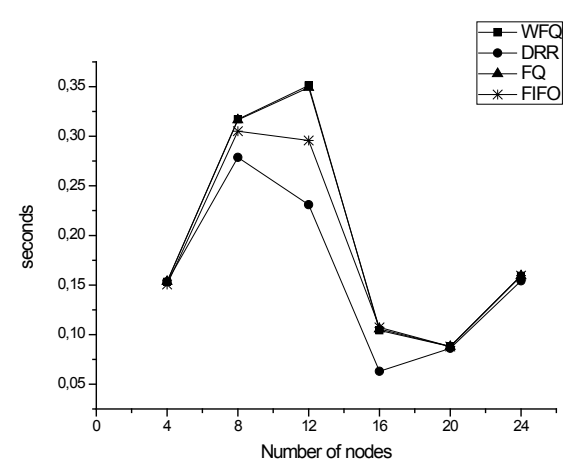

Figure 6. Average Delay for BE

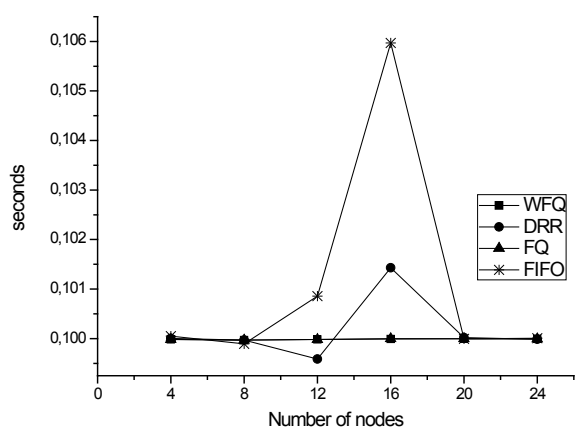

Figure 7. Average Jitter for UGS

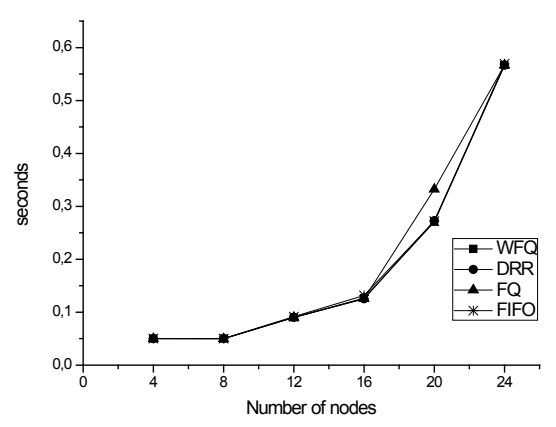

Figure 8. Average Jitter for rtPS

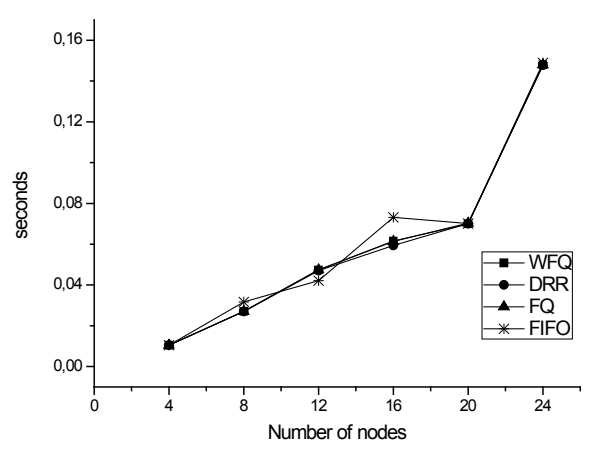

Figure 9. Average Jitter for nrtPS

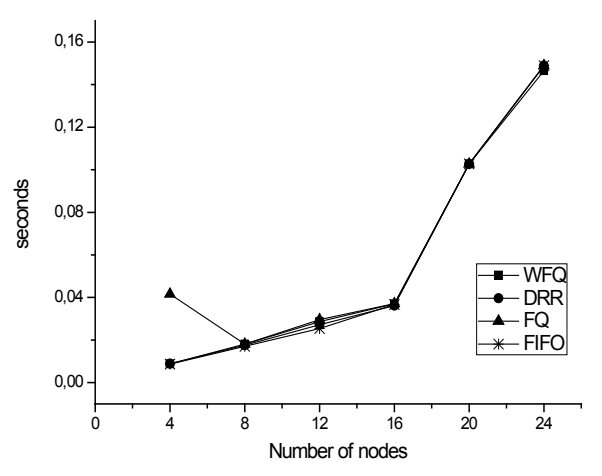

Figure 10. Average Jitter for BE 


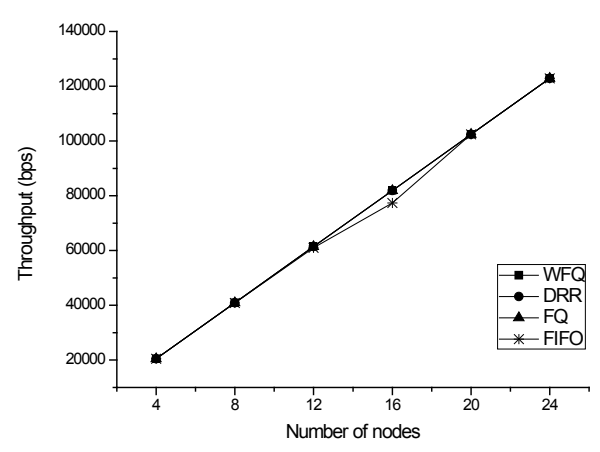

Figure 11. Average throughput for UGS

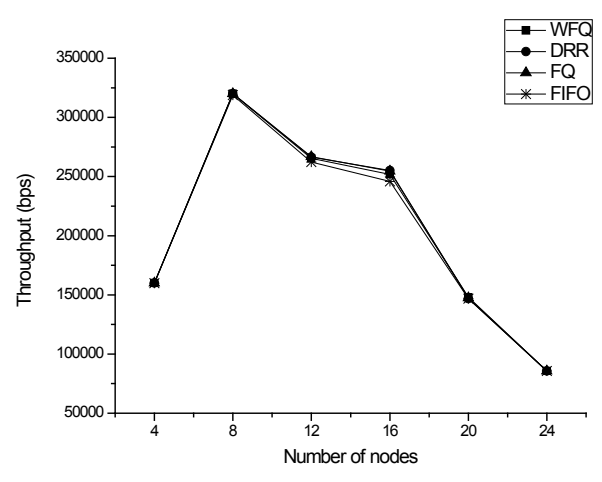

Figure 12. Average throughput for rtPS

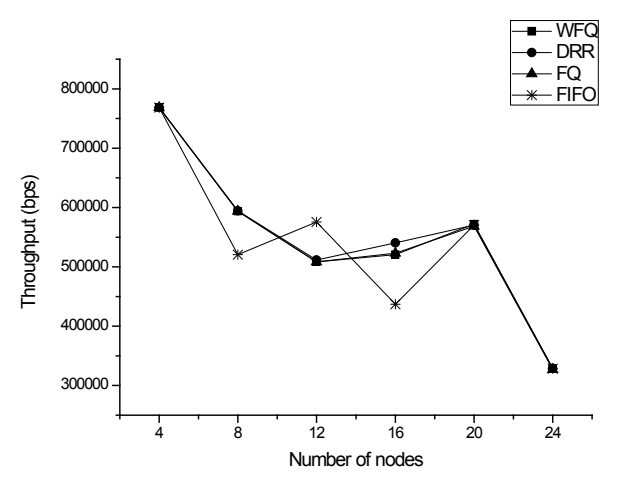

Figure 13. Average throughput for nrtPS

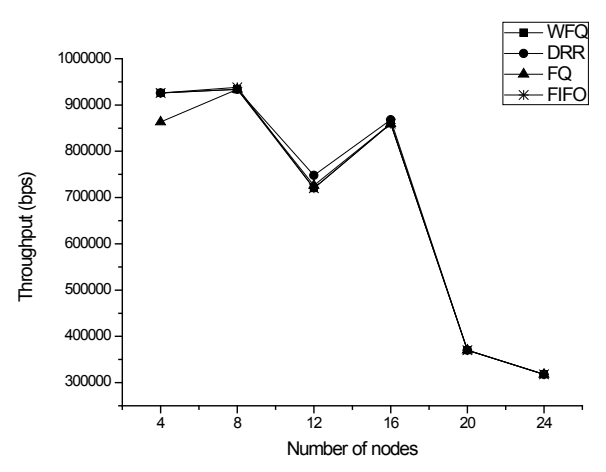

Figure 14. Average throughput for BE
Figures 7, 8, 9 and 10 show that the jitter is minimal when the network is not loaded, increasing the number of nodes causes an increase of the jitter. This could be explained by the increase of delay in queues. The average jitter is important for rtPS, nrtPS and BE applications, also the low jitter is guaranteed using the WFQ algorithm especially for the UGS application.

Based on figure 11, 12, 13 and 14, there is a degradation of throughput depending on growth in the number of nodes, but it remains constant for UGS flows. The throughput for certain classes is relatively higher than other classes, because of the sizes of packets generated by the traffic generator and the throughput designed to each application. All presented algorithm have the same behavior to guarantee the throughput so if the network is congested only the UGS flows are not affected. This proposed architecture guarantees all parameters that manage QoS for UGS flows using the WFQ algorithm.

$\rightarrow$ The performance evaluation of the WFQ algorithm

These scenarios present the performance evaluation of the WFQ algorithm. The average delay depends on network state (number of nodes) is presented in figure 15 . The average delay of UGS flows remains constant, while nrtPS flow delay reaches a peak 1,173 (s) and a slight increase in delay for the rtPS stream. This is due to the increase in numbers of received packets of each flow. As soon as the number of nodes exceeds 16 , the average delay decreases, this reflects the reduction in the number of packets received after the onset of congestion in queues (figure 18). This reduction is the result of a request for throughput reduction at sources. The great number of nodes which represent the network congestion, affects the behavior of jitter in rtPS, nrtPS and BE flows and then a steady state for the UGS flow (figure 16), this could be explained by the added delay in the queues caused by congestion. The performance in terms of average throughput of rtPS, nrtPS and BE flows is decreased due to the growth of the number of nodes, but the average throughput of UGS flows remains constant (figure 17).

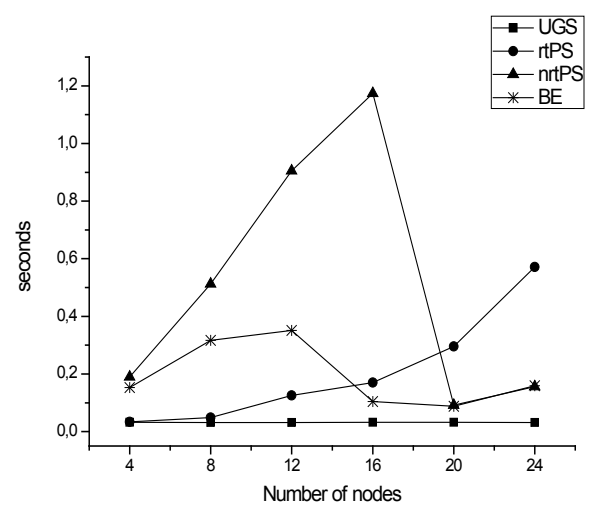

Figure 15. Average delay for WFQ 


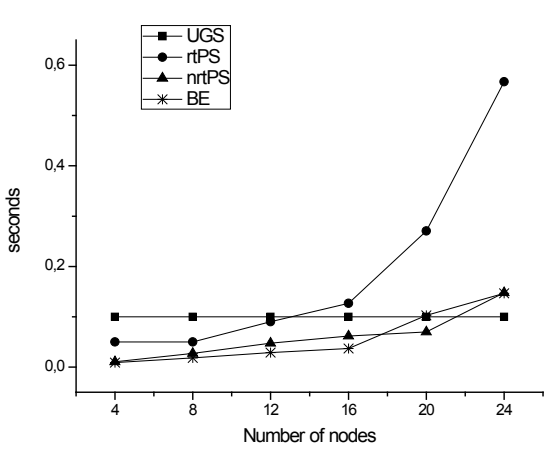

Figure 16. Average jitter for WFQ

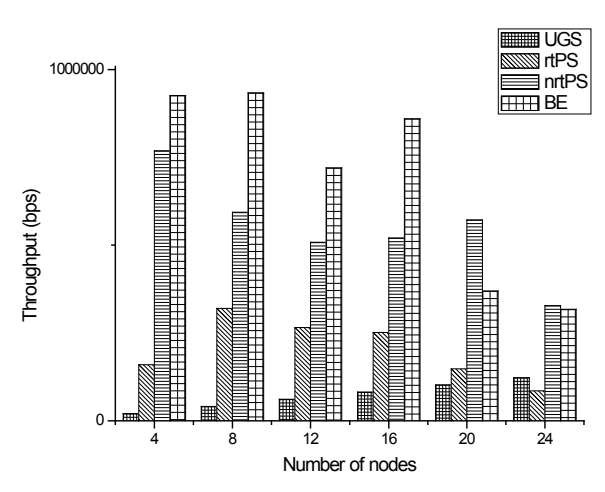

Figure 17. Average throughput for WF

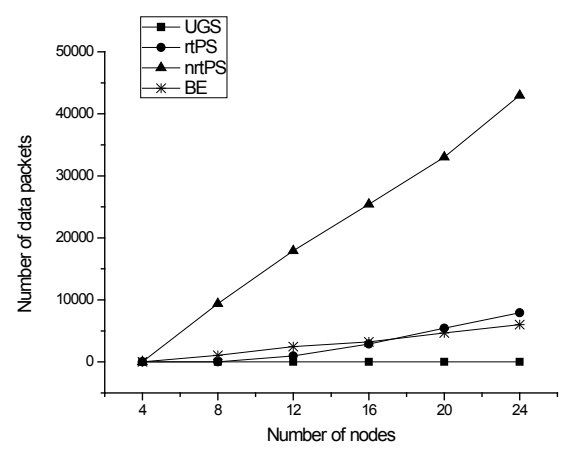

Figure 18. Variation Packet loss for WFQ

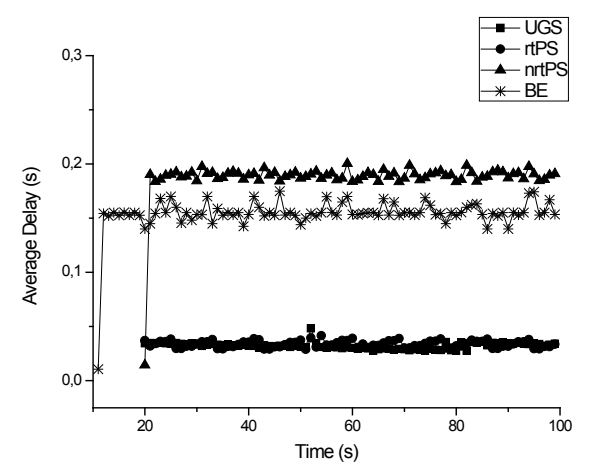

Figure 19. Variation average delay for WFQ

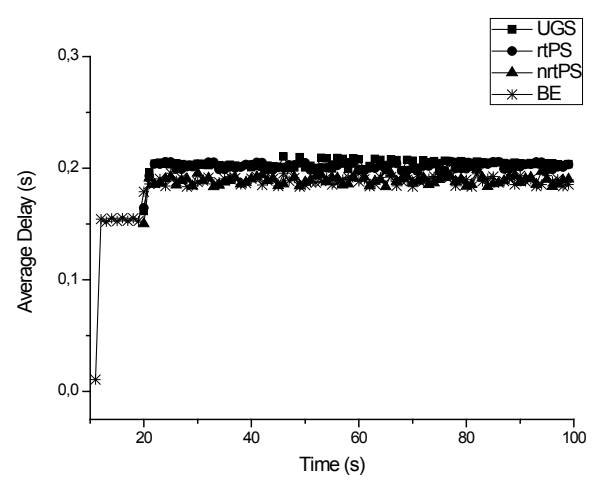

Figure 20. Variation average delay for FIFO

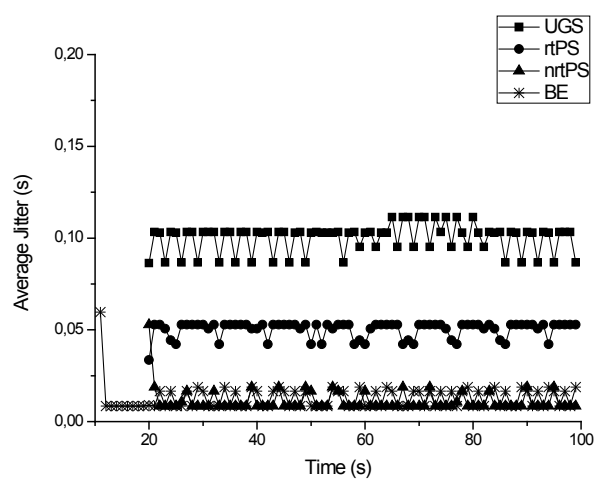

Figure 21. Variation average jitter for WFQ

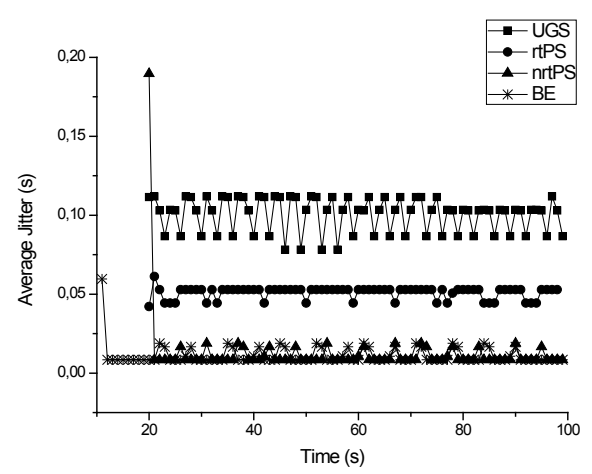

Figure 22. Variation average jitter for FIFO

The variation of delay for various flows according to time in fixed number of nodes is almost constant and identical. The figure 19 presents a differentiation in delay between flows. BE delay increases from time $t=20(\mathrm{~s})$, this presents the network behavior in the case of saturation that respects constraints of other service classes using WFQ algorithm. This variation starts at the transmission of UGS, rtPS and nrtPS flows. Figure 21 presents that the jitter remains stable for different flows. Figure 20 and 22 describe the network behavior to respect constraints (jitter and delay) using FIFO algorithm.

This paper, presents results of simulation concerning the parameter of QoS for various techniques of scheduling in 
WiMAX network. After analyzing the simulation results which shows the variations of delay, throughput, jitter and packet received. FIFO algorithm has the same behavior to respect QoS to all service classes without differentiation of service, also the other algorithm guarantee required constraints of different flows. But this guarantee is limited to the DRR and FQ in not congested networks, so if the number of nodes increases, it affects QoS. As well, these results show that the WFQ algorithm ensures good quality of services especially for multimedia applications. It maintains low latency for applications with high temporal constraints.

\section{Conclusions}

The rapid evolution of communications systems and the growing needs for users to multimedia applications require the QoS guarantee to each application. Especially, in wireless networks pose special problems needs hard mechanisms that support QoS. This work presents the various parameters characterizing the network performance and the implementation of mechanisms that manage these parameters such as scheduling, buffer management and flow classification. In this paper, we presented the performance evaluation of scheduling algorithm in WiMAX network using the NS2 simulator. These simulations describe the behavior of each algorithm to respect the QoS of each service class. The studied parameters have a direct influence on the flows nature such as the delay, throughput, jitter and loss rate. We conclude that WFQ algorithm guarantee the QoS of each service class in WiMAX networks. This algorithm provides required parameters to each application also when the network is congested.

The proposed design was analyzed: simulation results show a significant performance improvement in terms of overall throughput and delay when compared to recently published work. It will be described in VHDL language at RTL levels to be synthesized in a low level.

\section{REFERENCES}

[1] IEEE 802.16-2004, IEEE Standard for Local and metropolitan area networks, Part 16: Air interface For Fixed Broadband Wireless Access Systems, October 2004

[2] IEEE Std 802.16g-2007, IEEE Standard for Local and metropolitan area networks, Part 16: Air Interface for Fixed and Mobile Broadband Wireless Access Systems, Amendment 3: Management Plane Procedures and Services, December 2007 (Approved: 27 September 2007)

[3] Rohit A. Talwalkar and Mohammad Ilyas, "Analysis of Quality of Service (QoS) in WiMAX networks", in: Proceeding of the 16th IEEE International Conference on Networks, December 2008, pp. 1-8

[4] Aymen Belghith and Loutfi Nuaymi, "Design and implementation of a QoS-included WIMAX Module for NS-2 Simulator", in: Proceedings of the 1st international confe- rence on Simulation tools and techniques for communications, networks and systems \& workshops, Article No. 28, 2008. (Marseille, France)

[5] Jin-Cherng Lin, Chun-Lun Chou and Cheng-Hsiung Liu, "Performance Evaluation For Scheduling Algorithms In WiMAX Network", in: Proceeding of the 22th International Conference on, Advanced Information Networking and Application, (AINA Workshops 2008), March 2008, pp. 68-74

[6] Najah Abu Ali, Pratik Dhrona and Hossam Hassanein, "A performance study of uplink scheduling algorithms in point-to-multipoint WiMAX networks", Computer Communications, Volume 32 Issue 3 February 2009, pp. 511-521. (Butterworth-Heinemann).

[7] Mikael Gidlund and Gang Wang, "Uplink Scheduling Algorithms for QoS Support in Broadband Wireless Access Networks", in: Proceeding of the Journal of Communications, Volume 4, No 2, March 2009, pp. 133-142

[8] Juliana Freitag and Nelson L.S. da Fonseca, "Uplink Scheduling with Quality of Service in IEEE 802.16 Networks", in: Proceeding of the Global Telecommunications Conference (GLOBECOM '07.IEEE), November 2007, pp. 2503-2508 (Washington)

[9] K. Yamakoshi, K. Nakai, E. Oki and N. Yamanaka, "Dynamic deficit round-robin scheduling scheme for variable-length packets", in: IEEE Electronics Letters, Volume 38, Issue 3, 31 Jan 2002, pp.148 - 149

[10] Haitham Tayyar and Hussein Alnuweiri, "The Complexity of Computing Virtual-Time in Weighted Fair Queuing Schedulers", in: IEEE International Conference on Communications, Volume 4, 20-24 June 2004, pp. 1996 - 2002

[11] Andrea Francini and Fabio M. Chiussi, "A Weighted Fair Queueing Scheduler with Decoupled Bandwidth and Delay Guarantees for the Support of Voice Traffic", IEEE, Global Telecommunications Conference, Volume 3, 25-29 Nov. 2001, pp. $1821-1827$

[12] The network simulator-ns-2. http://www.isi.edu/nsnam/ns/. Page accessed on April. 2009

[13] Juliana Freitag and Nelson L. S. da Fonseca, "Wimax Module For The NS-2 Simulator", in: Proceeding of the 18th IEEE International Symposium on Personal Indoor and Mobile Radio Communications, September 2007, pp. 1-6

[14] Frank Chee-Da Tsai, Jenhui Chen, Chiang-Wei Chang, Wei-Jen Lien, Chih-Hsin Hung and Jui-Hsiang Sum, "The Design and Implementation of WiMAX Module for ns-2 Simulator", WNS2 '06: Proceedings from the 2006 Workshop on ns-2: the IP network simulator, Octobre 2006

[15] National Institute of Standards and Technology, "The Network Simulator NS-2 NIST add-on IEEE 802.16 model (MAC+PHY)", National Institute of Standards and Technology - Draft 1.2.1, January 2009, pp. 1-29 